16

\title{
Проектирование конструкций нейтральных неполяризующих интерференционных систем, размещенных между средами с одинаковыми показателями преломления
}

\author{
(C) В.Х. Фам, Т.Ф. Нго, Л.А. Губанова \\ Университет ИТМО, \\ 197101 Санкт-Петербург, Россия \\ e-mail: phamhoa0303@mail.ru
}

Поступила в редакцию 21.10.2019 г.

В окончательной редакции 21.10.2019 г.

Принята к публикации 28.10.2019 г.

Рассмотрено проектирование конструкций нейтральных неполяризующих интерференционных систем, сформированных на грани одной из двух призм, которые являются составляющими оптического элемента в виде куба. Проведен анализ спектральных характеристик энергетического коэффициента отражения разработанных конструкций.

Ключевые слова: интерференционные системы, поляризация, неполяризующие светоделители, нейтральные фильтры, стеклянные кубы.

DOI: $10.21883 / O S .2020 .02 .48977 .285-19$

\section{Введение}

В оптических системах нередко встречаются элементы, созданные соединением двух призм. Такая склейка представляет собой некий параллелепипед. Наиболее часто в оптическом приборостроении используются параллелепипеды в виде куба. Подобные кубы создаются из двух прямоугольных призм, имеющих одинаковую форму и размеры, изготовленных из материалов с одинаковыми показателями преломления. Такие кубы часто используются в оптических системах для разделения излучения на две части: отраженную и прошедшую [1]. Разделение излучения происходит на гипотенузной грани двух соединенных призм. В этом случае излучение падает на границу соединения призм под некоторым наклонным углом, обычно $45^{\circ}$. Это приводит к появлению поляризации отраженного (прошедшего) излучения, т. е. коэффициент отражения (пропускания) состоит из двух составляющих $s$ - и $p$-компонент [2]. Однако в ряде случаев необходимо разделить излучение по интенсивности без деления излучения на $s$ - и $p$-поляризации [3]. С этой целью на гипотенузной грани одной из двух соединенных призм формируется нейтральная неполяризующая интерференционная система (ННИС). Очевидно, что при этом оптическое покрытие размещается между средами с одинаковыми показателями преломления, равными показателю преломления материала, из которого изготавливаются призмы (рис. 1). Кубы, в которых формируется ННИС, называются неполяризующими светоделителями (НС). В последние годы НС входят в состав многих интерферометров, используемых для измерения различных величин с высокой точностью $[4,5]$.

Спектральные характеристики ННИС должны удовлетворять двум требованиям: 1) незначительная разни- ца между спектральными характеристиками отражения (пропускания) для $s$ - и $p$-поляризаций, т.е. $R_{s} \approx R_{p}$, $\left.T_{s} \approx T_{p} ; 2\right)$ постоянство отношения отражения к пропусканию, т. е. $T / R=$ const.

Задача проектирования конструкций ННИС давно привлекает внимание разработчиков. Баумейстер (1961) и Костич (1970) первыми предложили теорию неполяризующих интерференционных систем, слои которых изготавливаются из двух пленкообразующих материалов, сформированных на плоскопараллельной пластинке [6,7]. Далее, Телен (1976) изложил метод поиска конструкций неполяризующих фильтров, основанных на использовании трех или более пленкообразующих материалов [8]. В ближайшее время применение численных методов позволяет создать различные методики поиска конструкций ННИС. Разработанные конструкции опубликованы в ряде работ [9-14]. Большинство предложенных конструкций были сформированы на плоскопараллельной пластинке, т. е. интерференционные системы размещены между средами с разными показателями преломления (воздух-стекло). Проектирование конструкций ННИС, размещенных между средами с одинаковыми показателями преломления, является более трудным для обеспечения всех указанных требований ННИС в широком диапазоне спектра $[13,14]$. Недостатком приведенных конструкций является то, что их спектральные характеристики не могут обеспечить малое расхождение между двумя поляризациями при постоянном отношении $T / R$ в широком диапазоне длин волн, или их структура достаточна сложна, в состав входит большое количество слоев, что приводит к трудности в массовом производстве.

В настоящей работе рассмотрено проектирование конструкций ННИС, размещенных между средами с одина- 


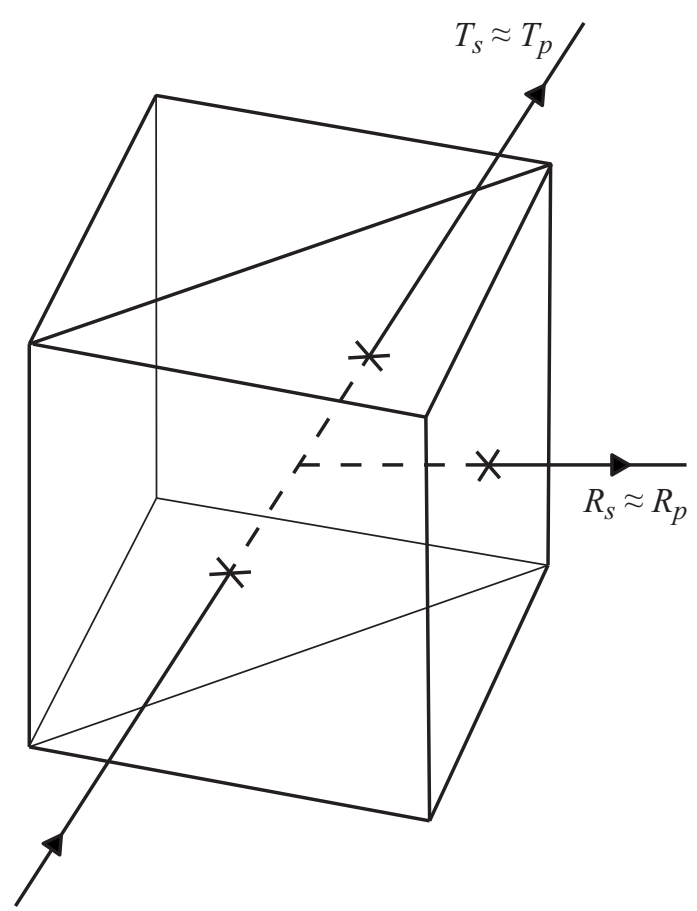

Рис. 1. Схема работы нейтрального неполяризующего интерференционного покрытия в стеклянном кубе.

ковыми показателями преломления, при этом расхождение между двумя поляризациями не превышает 2\%, и отношение $T / R$, близкое к единице, сохраняется в интервале длин волн $\Delta \lambda \geq 50 \mathrm{~nm}$.

Проектирование конструкций ННИС может быть разделено на два этапа: первый, определение показателей преломления пленкообразующих материалов, из которых формируются слои; второй, определение количества слоев и их толщин.

\section{Определение пленкообразующих материалов, необходимых для формирования слоев ННИС}

Определение пленкообразующих материалов, из которых формируются слои, осуществляется путем установки соотношения между их показателями преломления так, чтобы расхождение между спектральными характеристики отражения (пропускания) для $s$ и $p$-поляризаций было минимальным. Согласно [15] при ограничении на толщины слоев (четвертьволновые слои) для интерференционной системы, состоящей из $N$ слоев, изготовленных из пленкообразующих материалов с показателями преломления $n_{1}, n_{2}, \ldots, n_{N}$, размещенной между средами с показателями преломления $n_{0}$ и $n_{m}$, можно представить следующие соотношения:

$$
\begin{aligned}
& \left(\Delta n \Delta n_{m}\right)^{1 / 2}\left(\Delta n_{2} \Delta n_{4} \Delta n_{6} \ldots \Delta n_{2 k}\right)= \\
& =\Delta n_{1} \Delta n_{3} \Delta n_{5} \ldots \Delta n_{2 k+1}
\end{aligned}
$$

в случае, когда количество слоев является нечетным $N=2 k+1$, и

$$
\begin{aligned}
& \left(\Delta n_{0} / \Delta n_{m}\right)^{1 / 2}\left(\Delta n_{2} \Delta n_{4} \Delta n_{6}\right) \ldots \Delta n_{2},= \\
& =\Delta n_{1} \Delta n_{3} \Delta n_{5} \ldots \Delta n_{2 k-1}
\end{aligned}
$$

в случае, когда количество слоев является четным $(N=2 k)$, где $\Delta n_{j}$ - отношение показателя преломления $j$-го слоя для $s$-поляризации к его показателю преломления для $p$-поляризации $(j=1,2, \ldots, N)$ и определяется следующим выражением:

$$
\Delta n_{j}=n_{j, s} / n_{j, p}=1 /\left(1-\left(n_{0} \sin \alpha_{0} / n_{j}\right)^{2}\right),
$$

где $\alpha_{0}-$ угол падения излучения на систему.

В случае, когда интерференционная система размещается между средами с одинаковыми показателями преломления $\left(n_{0}=n_{m}\right)$ и ее слои изготавливаются из трех пленкообразующих материалов с показателями преломления $n_{L}, n_{M}$ и $n_{H}$, соотношения (1)-(3) имеют вид

$$
\Delta n_{m}\left(\Delta n_{M}\right)^{k}=\left(\Delta n_{L}\right)^{[k / 2]+1}\left(\Delta n_{H}\right)^{[k / 2]}
$$

в случае, когда количество слоев является нечетным, и

$$
\left(\Delta n_{M}\right)^{k}=\left(\Delta n_{L}\right)^{[k / 2+1 / 2]}\left(\Delta n_{H}\right)^{[k / 2]}
$$

в случае, когда количество слоев является четным. Обозначение $[x]$ используется для операции выделения целой части вещественного числа $x$. Например, $[4,5]=4$ :

$$
\Delta n_{j}=n_{j, s} / n_{j, p}=1\left(1-\left(n_{m} \sin \alpha_{0} / n_{j}\right)^{2}\right) .
$$

Пусть $n_{L}=1.38\left(\mathrm{MgF}_{2}\right), \quad n_{H}=2.35\left(\mathrm{TiO}_{2}\right) \quad$ и $\quad n_{m}=$ $=1.52($ К8). Задача определения пленкообразующих материалов, из которых формируются слои ННИС, сводится к определению показателя преломления материала $n_{\mathrm{M}}$ с использованием выражений (4)-(6). На основе приведенных выражений можно представить связь между показателем преломления $n_{M}$ и количеством слоев $N$ в графическом виде, как показано на рис. 2. Видно, что при суммировании обоих случаев, когда количество слоев нечетное (кривая 1) и четное (2), значение показателя преломления $n_{M}$ лежит в интервале $1.57-1.67$. Из

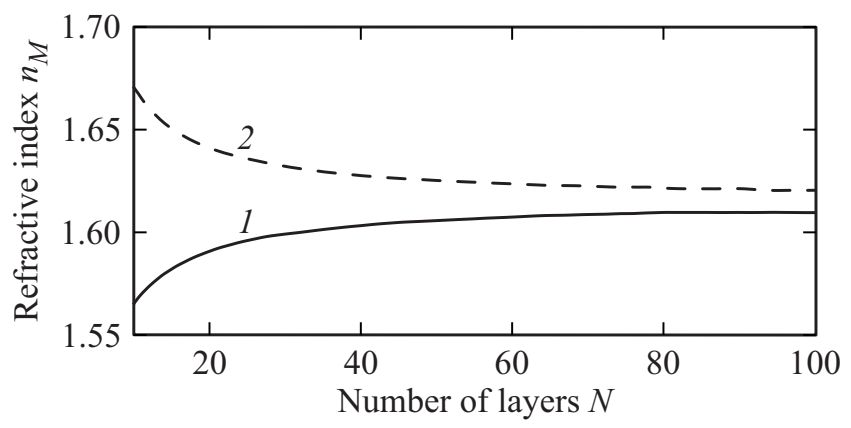

Рис. 2. Зависимость показателя преломления слоев $M$ от количества слоев $N$, когда количество слоев, входящих в систему, является нечетным (1) и четным (2). 
набора распространенных пленкообразующих материалов можно выбрать оксид алюминия $\mathrm{Al}_{2} \mathrm{O}_{3}\left(n_{M}=1.65\right)$ в качестве пленкообразующего материала слоев, имеющего средний показатель преломления. В результате были определены следующие показатели преломления слоев ННИС: $n_{L}=1.38\left(\mathrm{MgF}_{2}\right), n_{M}=1.65\left(\mathrm{Al}_{2} \mathrm{O}_{3}\right)$ и $n_{H}=2.35\left(\mathrm{TiO}_{2}\right)$.

Аналогично в случае, когда слои, входящие в состав ННИС, изготавливаются из четырех пленкообразующих материалов, были определены следующие показатели преломления: $n_{L}=1.38\left(\mathrm{MgF}_{2}\right), n_{M 1}=1.45\left(\mathrm{SiO}_{2}\right)$, $n_{M 2}=1.65\left(\mathrm{Al}_{2} \mathrm{O}_{3}\right)$ и $n_{H}=2.35\left(\mathrm{TiO}_{2}\right)$.

\section{Определение количества слоев и их оптических толщин}

Определение количества слоев и их толщин выполняется с помощью численного метода, основанного на использовании некой целевой функции. В настоящей работе целевая функция (Merit Function, MF) имеет вид

$$
M F=\frac{1}{2 \mu} \sum_{i=1}^{L}\left[\frac{\left(R_{i, s}^{C}-R_{i, s}^{G}\right)^{2}}{\varepsilon_{i, s}^{2}}+\frac{\left(R_{i, p}^{C}-R_{i, p}^{G}\right)^{2}}{\varepsilon_{i, p}^{2}}\right]^{1 / 2}
$$

где $\mu$ - количество точек в шкале длин волн, $R_{i, s}^{C}$ и $R_{i, p}^{C}$ - расчетное значение коэффициентов отражения, $R_{i, s}^{G}$ и $R_{i, p}^{G}$ - целевое значение коэффициентов отражения, $\varepsilon_{i, s}$ и $\varepsilon_{i, p}$ - допустимое отклонение для $s$ - и $p$-поляризаций.

Задача определения количества слоев и их толщин сводится к минимизации приведенной целевой функции, т.е. поиск значений количества слоев и их толщин, так, чтобы значение целевой функции было минимальным. С другой стороны, спектральные характеристики энергетического коэффициента отражения полученных конструкций должны удовлетворять ряду следующих заданных условий:

$$
\begin{gathered}
\left|R_{s}-R_{p}\right|<2 \%, \\
\left|R_{s}-50 \%\right|<2 \%, \\
\left|R_{p}-50 \%\right|<2 \%, \\
\Delta \lambda \geq 50 \mathrm{~nm} .
\end{gathered}
$$

В начале процесса минимизации необходимо вводить некоторую начальную ячейку. Начальная ячейка должна иметь малое расхождение между двумя поляризациями в диапазоне спектра $\Delta \lambda \geq 50 \mathrm{~nm}$. В настоящей работе конструкция вида (НСВС) ${ }^{6} \mathrm{H}$ была выбрана в качестве начальной ячейки, поскольку она имеет незначительное расхождение между двумя поляризациями в диапазоне длин волн 460-540 nm. В результате процесса минимизации была получена некая конструкция, структура которой показана в табл. 1. Все полученные оптические толщины могут быть реализованы на практике при
Таблица 1. 30-слойная система, изготовленная из фторида магния $\left(\mathrm{MgF}_{2}\right)$, оксида алюминия $\left(\mathrm{Al}_{2} \mathrm{O}_{3}\right)$ и оксида титана $\left(\mathrm{TiO}_{2}\right), \lambda_{0}=550 \mathrm{~nm}$

\begin{tabular}{c|c|c|c|c|c}
\hline $\begin{array}{c}\text { No } \\
\text { слоя }\end{array}$ & $\begin{array}{c}\text { Мате- } \\
\text { риал }\end{array}$ & $\begin{array}{c}\text { Оптическая } \\
\text { толщина } \lambda_{0}\end{array}$ & $\begin{array}{c}\text { № } \\
\text { слоя }\end{array}$ & $\begin{array}{c}\text { Мате- } \\
\text { риал }\end{array}$ & $\begin{array}{c}\text { Оптическая } \\
\text { толщина } \lambda_{0}\end{array}$ \\
\hline & $\mathrm{K} 8$ & & & & \\
\hline 1 & $\mathrm{MgF}_{2}$ & 0.40 & 16 & $\mathrm{TiO}_{2}$ & 0.25 \\
2 & $\mathrm{Al}_{2} \mathrm{O}_{3}$ & 1.00 & 17 & $\mathrm{Al}_{2} \mathrm{O}_{3}$ & 0.3 \\
3 & $\mathrm{TiO}_{2}$ & 0.25 & 18 & $\mathrm{TiO}_{2}$ & 0.20 \\
4 & $\mathrm{MgF}_{2}$ & 0.30 & 19 & $\mathrm{MgF}_{2}$ & 0.10 \\
5 & $\mathrm{TiO}_{2}$ & 0.15 & 20 & $\mathrm{Al}_{2} \mathrm{O}_{3}$ & 0.30 \\
6 & $\mathrm{Al}_{2} \mathrm{O}_{3}$ & 0.15 & 21 & $\mathrm{MgF}_{2}$ & 0.20 \\
7 & $\mathrm{TiO}_{2}$ & 0.30 & 22 & $\mathrm{TiO}_{2}$ & 0.15 \\
8 & $\mathrm{MgF}_{2}$ & 0.20 & 23 & $\mathrm{Al}_{2} \mathrm{O}_{3}$ & 0.35 \\
9 & $\mathrm{Al}_{2} \mathrm{O}_{3}$ & 0.20 & 24 & $\mathrm{TiO}_{2}$ & 0.30 \\
10 & $\mathrm{TiO}_{2}$ & 0.15 & 25 & $\mathrm{Al}_{2} \mathrm{O}_{3}$ & 0.25 \\
11 & $\mathrm{MgF}_{2}$ & 0.25 & 26 & $\mathrm{MgF}_{2}$ & 0.45 \\
12 & $\mathrm{Al}_{2} \mathrm{O}_{3}$ & 0.30 & 27 & $\mathrm{Al}_{2} \mathrm{O}_{3}$ & 0.35 \\
13 & $\mathrm{MgF}_{2}$ & 0.25 & 28 & $\mathrm{TiO}_{2}$ & 0.18 \\
14 & $\mathrm{TiO}_{2}$ & 0.10 & 29 & $\mathrm{Al}_{2} \mathrm{O}_{3}$ & 0.45 \\
15 & $\mathrm{Al}_{2} \mathrm{O}_{3}$ & 0.35 & 30 & $\mathrm{MgF}_{2}$ & 0.40 \\
\hline & & & & $\mathrm{K} 8$ &
\end{tabular}

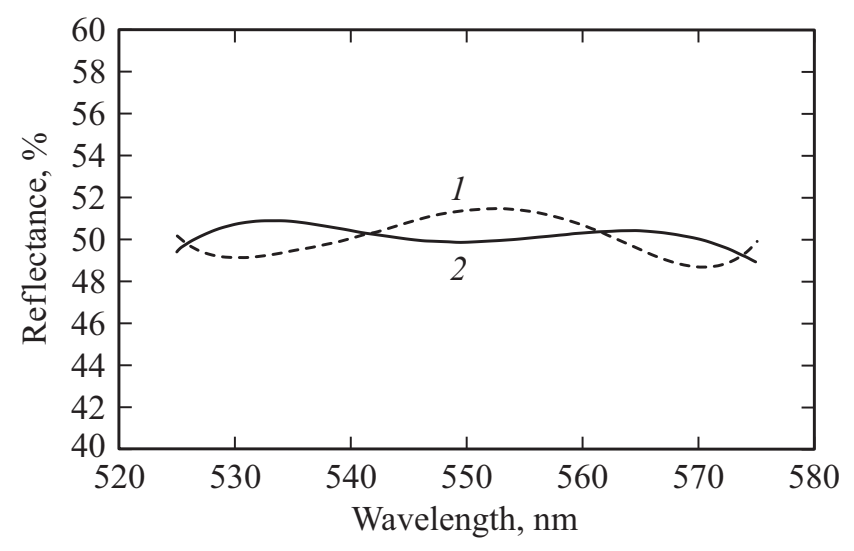

Рис. 3. Спектральные характеристики энергетического коэффициента отражения 30-слойной ННИС для $s$ - поляризации (1) и $p$-поляризации $(2), \alpha_{0}=45^{\circ}, \lambda_{0}=550 \mathrm{~nm}$.

использовании существующих методов формирования оптических покрытий.

На рис. 3 представлены спектральные характеристики отражения разработанной интерференционной системы, состоящей из 30 слоев. Видно, что значение энергетического коэффициента отражения для $s$ - и $p$-поляризаций лежит в интервале $(50 \pm 2) \%$ в диапазоне длин волны (525-575) nm, что соответствует условию (7). Разработанная 30-слойная система является примером ННИС с диапазоном работы $\Delta \lambda=50 \mathrm{~nm}$.

Расширение диапазона работы ННИС может быть осуществлено увеличением количества слоев, входящих в состав системы или увеличением количества пленко- 
Таблица 2. 34-слойная система, изготовленная из фторида магния $\left(\mathrm{MgF}_{2}\right)$, оксида алюминия $\left(\mathrm{Al}_{2} \mathrm{O}_{3}\right)$ и оксида титана $\left(\mathrm{TiO}_{2}\right), \lambda_{0}=550 \mathrm{~nm}$

\begin{tabular}{c|c|c|c|c|c}
\hline $\begin{array}{c}\text { No } \\
\text { слоя }\end{array}$ & $\begin{array}{c}\text { Мате- } \\
\text { риал }\end{array}$ & $\begin{array}{c}\text { Оптическая } \\
\text { толщина } \lambda_{0}\end{array}$ & $\begin{array}{c}\text { № } \\
\text { слоя }\end{array}$ & $\begin{array}{c}\text { Мате- } \\
\text { риал }\end{array}$ & $\begin{array}{c}\text { Оптическая } \\
\text { толщина } \lambda_{0}\end{array}$ \\
\hline & $\mathrm{K} 8$ & & & & \\
\hline 1 & $\mathrm{MgF}_{2}$ & 0.2250 & 18 & $\mathrm{TiO}_{2}$ & 0.2750 \\
2 & $\mathrm{Al}_{2} \mathrm{O}_{3}$ & 0.5000 & 19 & $\mathrm{Al}_{2} \mathrm{O}_{3}$ & 0.3250 \\
3 & $\mathrm{MgF}_{2}$ & 0.4500 & 20 & $\mathrm{TiO}_{2}$ & 0.1875 \\
4 & $\mathrm{Al}_{2} \mathrm{O}_{3}$ & 0.2500 & 21 & $\mathrm{MgF}_{2}$ & 0.1500 \\
5 & $\mathrm{TiO}_{2}$ & 0.2750 & 22 & $\mathrm{Al}_{2} \mathrm{O}_{3}$ & 0.3000 \\
6 & $\mathrm{MgF}_{2}$ & 0.3250 & 23 & $\mathrm{MgF}_{2}$ & 0.1375 \\
7 & $\mathrm{TiO}_{2}$ & 0.1550 & 24 & $\mathrm{TiO}_{2}$ & 0.2000 \\
8 & $\mathrm{Al}_{2} \mathrm{O}_{3}$ & 0.1650 & 25 & $\mathrm{Al}_{2} \mathrm{O}_{3}$ & 0.3250 \\
9 & $\mathrm{TiO}_{2}$ & 0.2500 & 26 & $\mathrm{TiO}_{2}$ & 0.2750 \\
10 & $\mathrm{MgF}_{2}$ & 0.1700 & 27 & $\mathrm{Al}_{2} \mathrm{O}_{3}$ & 0.3250 \\
11 & $\mathrm{Al}_{2} \mathrm{O}_{3}$ & 0.2000 & 28 & $\mathrm{MgF}_{2}$ & 0.4000 \\
12 & $\mathrm{TiO}_{2}$ & 0.1750 & 29 & $\mathrm{Al}_{2} \mathrm{O}_{3}$ & 0.3125 \\
13 & $\mathrm{MgF}_{2}$ & 0.2400 & 30 & $\mathrm{TiO}_{2}$ & 0.1375 \\
14 & $\mathrm{Al}_{2} \mathrm{O}_{3}$ & 0.3000 & 31 & $\mathrm{MgF}_{2}$ & 0.0875 \\
15 & $\mathrm{MgF}_{2}$ & 0.2250 & 32 & $\mathrm{TiO}_{2}$ & 0.1000 \\
16 & $\mathrm{TiO}_{2}$ & 0.1250 & 33 & $\mathrm{Al}_{2} \mathrm{O}_{3}$ & 0.3250 \\
17 & $\mathrm{Al}_{2} \mathrm{O}_{3}$ & 0.3250 & 34 & $\mathrm{MgF}_{2}$ & 0.4000 \\
\hline & & & & $\mathrm{K} 8$ & \\
\hline & & & & &
\end{tabular}

образующих материалов, из которых формируются слои ННИС.

В табл. 2 представлена структура ННИС, состоящая из 34 слоев. Спектральные характеристики энергетического коэффициентов отражения для $s$ - и $p$-поляризаций показаны на рис. 4. Рассматриваемая конструкция обеспечивает условие (7) в интервале длин волн $520-580 \mathrm{~nm}$, т.е. $\Delta \lambda=60 \mathrm{~nm}$. Для иллюстрации влияния увеличения количества пленкообразующих материалов рассмотрена 46-слойная конструкция ННИС, изготовленная из четырех пленкообразующих материалов $\mathrm{MgF}_{2}(\mathrm{~L}), \mathrm{SiO}_{2}(\mathrm{M} 1), \mathrm{Al}_{2} \mathrm{O}_{3}(\mathrm{M} 2)$ и $\mathrm{TiO}_{2}(\mathrm{H})$. Структура рассматриваемой конструкций приведена на рис. 5, $a$. Спектральные характеристики энергетического коэффициентов отражения для $s$ - и $p$-поляризаций показаны на рис. 5, $b$. Такая конструкция обеспечивает выполнение условия (7) в интервале длин волн 500-600 nm, т.е. $\Delta \lambda=100 \mathrm{~nm}$.

\section{Заключение}

В работе рассмотрено проектирование конструкций нейтральных неполяризующих интерференционных систем, размещенных между средами с одинаковыми показателями преломления. Приведено соотношение между показателями преломления, которое служит основной для выбора пленкообразующих материалов, из которых формируются слои ННИС. Представлено определение количества слоев и их толщин численным методом при

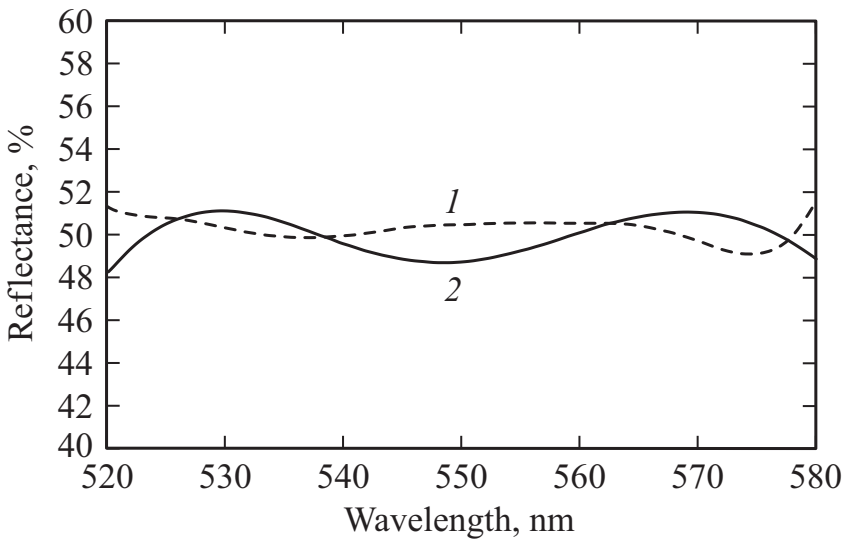

Pис. 4. Спектральные характеристики энергетического коэффициента отражения 34-слойной ННИС для $s$ - поляризации $(1)$ и $p$-поляризации $(2), \alpha_{0}=45^{\circ}, \lambda_{0}=550 \mathrm{~nm}$.
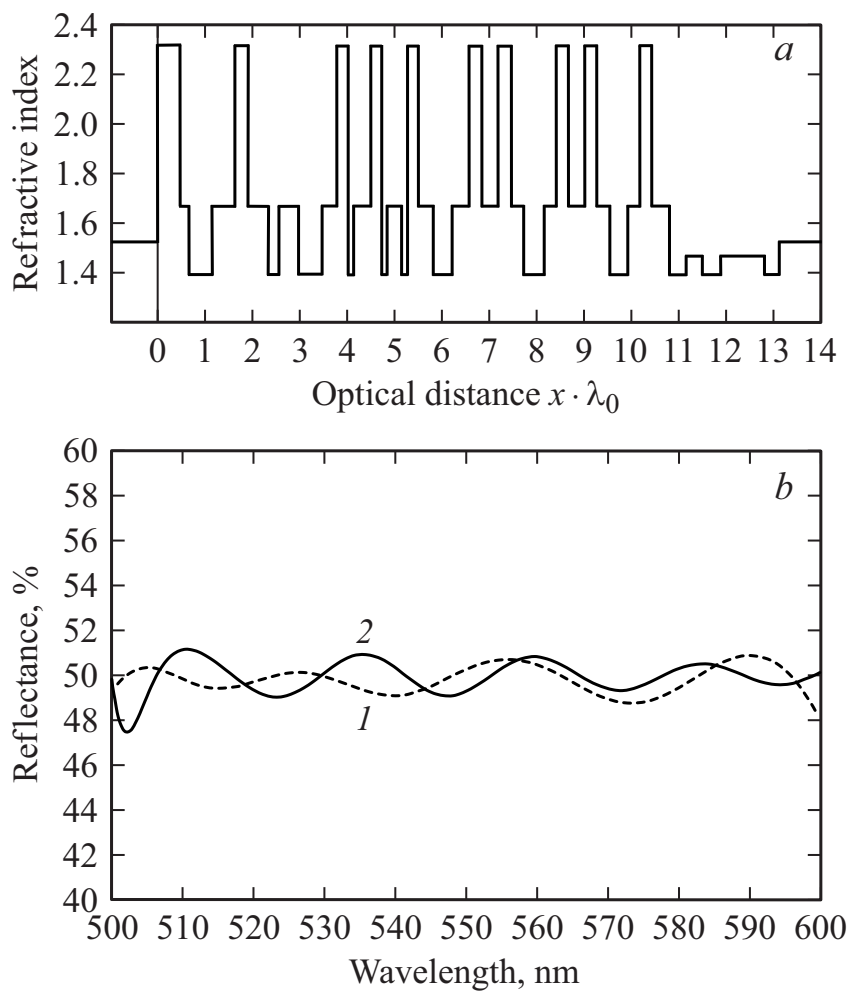

Рис. 5. (a) Распределение показателя преломления по оптической толщине 46-слойной ННИС из фторида магния $\mathrm{MgF}_{2}$, диоксида кремния $\mathrm{SiO}_{2}$ оксида алюминия $\mathrm{Al}_{2} \mathrm{O}_{3}$ и оксида титана $\mathrm{TiO}_{2}$, отнесенной к длине волны $\lambda_{0}=550 \mathrm{~nm}$. (b) Спектральные характеристики энергетического коэффициента отражения 46 -слойной ННИС для $s$ - (1) и $p$-поляризации (2), $\alpha_{0}=45^{\circ}$, $\lambda_{0}=550 \mathrm{~nm}$.

использовании целевой функции. Анализ спектральных характеристик полученных конструкций показал, что расхождение между двумя поляризациями не превышает $2 \%$ и отношение $T / R$ близко к единице в спектральном диапазоне $\Delta \lambda \geq 50 \mathrm{~nm}$. 


\section{Финансирование работы}

Работа выполнена при финансовой поддержке Министерства образования и науки Российской Федерации (Проект № 3.2506.2017/4.6).

\section{Конфликт интересов}

Авторы заявляют, что у них нет конфликта интересов.

\section{Список литературы}

[1] Baumeister P.W. Optical Coating Technology. SPIE. 2004. $840 \mathrm{p}$.

[2] Губанова Л.А., Путилин Э.С. Оптические покрытия. Учебник для вузов. СПб.: Лань, 2016. 286 с.

[3] Macleod A.H. Thin-Film Optical Filters, 4th Edition. CRC Press, 2010. $800 \mathrm{p}$.

[4] Lou Y., Yan L., Chen B., Zhang S. // Opt. Exp. 2017. V. 25. N 6. P. 6805-6821. doi 10.1364/OE.25.006805

[5] Niwa Y., Arai K., Ueda A., Sakagami M., Gouda N., Kobayashi Y., Yamada Y., Yano T. // Appl. Opt. 2009. V. 48. N 32. P. 6105-6110. doi 10.1364/AO.48.006105

[6] Baumeister P. // Opt. Act. 1961. V. 8. N 2. P. 105-119. doi 10.1080/713826378

[7] Costich V.R. // Appl. Opt. 1970. V. 9. N 4. P. 866-870. doi 10.1364/AO.9.000866

[8] Thelen A. // Appl. Opt. 1976. V. 15. N 12. P. 2983-2985. doi 10.1364/AO.15.002983

[9] Henderson A.R. // Thin Solid Films. 1978. V. 51. N 3. P. 339-347. doi 10.1016/0040-6090(78)90297-3

[10] Wang W., Xiong S., Zhang Y. // Appl. Opt. 2007. V. 46. N 16. P. 3185-3191. doi 10.1364/AO.46.003185

[11] Hongji Q., Ruijin H., Kui Y., Jianda S., Zhengxiu F. // Appl. Opt. 2005. V. 44. N 12. P. 2343-2348. doi 10.1364/AO.44.002343

[12] Tikhonravov A.V., Trubetskov M.K. // Proc. SPIE. 1994. V. 2253. P. 10-20.

[13] Jin H.S., Chun Y.G., Zheng P.W. // Appl. Opt. 2009. V. 48. N 18. P. 3385-3390. doi 10.1364/AO.48.003385

[14] Xu X., Shao J., Fan Z. // Appl. Opt. 2006. V. 45. P. 4297-4302.

[15] Фам В.Х., Нго Т.Ф., Губанова Л.А. // Комп. Опт. 2019. Т. 43. № 4. C. 550-556. doi 0.18287/2412-6179-2019-43-4-550-556 